Pecvnia, 4 (2007), pp. 1-25

\title{
La diversidad de género en el Código Unificado español y la práctica empresarial
}

\author{
Amalia Carrasco Gallego \\ agallego@us.es \\ Joaquina Laffarga Briones \\ quina@us.es \\ Universidad de Sevilla \\ Avda. Ramón y Cajal $n^{\circ} 1$ \\ 41018 Sevilla (Spain)
}

El 19 de mayo de 2006 se aprobó definitivamente el Código Unificado sobre Buen Gobierno Corporativo. Uno de los puntos que más se han debatido ha sido el relacionado con la diversidad en los consejos de administración. Bajo el principio de cumple o explica, las empresas que no consigan en 2007 la diversidad recogida en su articulado y en concreto la diversidad de genero, deberán explicar el motivo, así como los esfuerzos para mejorarla.

En este trabajo se justifica la necesidad de elaborar Códigos de Buen Gobierno, que la composición de los consejos de administración sea diversa y exista, en particular, diversidad de género como objetivo de eficiencia. Se comenta el trato que los Códigos emitidos por países europeos dan a la diversidad, para centrarnos en el Código Español. Adicionalmente, se pone de manifiesto la 19th of May of 2006 the Unified Code about Corporate Governance was definitely approved. One of the points more debated is the diversity in the boards of directors. With the "comply or explain" principle the company that will not obtain in 2007 the recommended diversity, and especially the diversity of genre, must explain the reason and the attempt of improving it.

This paper justifies the need to elaborate Corporate Governance Codes; that the composition of the boards of directors should be diverse, and especially that there is diversity of genre as objective of efficiency. The recommendations on diversity in the Codes emitted by European Countries are commented. And after, we centre on the Unified Code. 
situación real actual de las empresas españolas y por tanto, el desafío que supone para ellas el cumplimiento con el código.

Palabras clave: diversidad, gobiernos corporativos, consejos de administración, género.
Finally, it is revealed the real situation of the Spanish companies and therefore the challenge that supposes for them to comply with the Code.

Key words: Corporate governance, diversity, boards of directors, genre.

\section{INTRODUCCIÓN}

El 19 de mayo de 2006 se aprobó definitivamente el Código Unificado sobre buen Gobierno Corporativo. Tanto éste como el proyecto aprobado en Enero del mismo año, han sido y son duramente criticados. Uno de los puntos que más se han debatido ha sido el artículo 14 relacionado con la diversidad en los consejos de administración. Bajo el principio de cumple o explica, al que está sometido este código, las empresas que no consigan en 2007 la diversidad recogida en su articulado y en concreto la diversidad de genero en sus consejos de administración deberán explicar el motivo por el cuál no la cumplen así como los esfuerzos realizados para mejorar la diversidad de género en su seno.

Con esta comunicación se pretende justificar, en primer lugar la necesidad de elaborar Códigos de Buen Gobierno que sirvan de referencia en la gobernanza de las empresas. La necesidad de que la composición de los consejos de administración sea diversa en general y exista diversidad de género en particular como objetivo de eficiencia. Se pone de manifiesto el trato que los diversos Códigos de Buen Gobierno emitidos por los países europeos dan a la diversidad, para centrarnos en el trato que da a la diversidad el Código Español. Como complemento se comenta cual es la situación real actual de las empresas y por tanto el desafío que supone para ellas el cumplimiento con el código.

\section{NECESIDAD DE LOS CÓDIGOS DE BUEN GOBIERNOS}

Escándalos financieros recientes, como los de Enron (2001), World-Com (2002), o Parmalat (2003), han puesto, en tela de juicio las prácticas financieras de las empresas, provocando una pérdida de confianza del mercado en la información de las empresas cotizadas. Esta pérdida de confianza se produce por un fallo en el funcionamiento de los controles, que permite ocultar la verdadera situación financiera de las empresas, y que se traduce en una supervisión poco adecuada por parte de los consejos de 
administración, incorrecciones en la auditoria interna y control de riesgos, además de falta de transparencia informativa y una auditoria externa deficiente. $Y$ lo que es más, estos fallos pasan desapercibidos para analistas y agencias de calificación, con lo cual, la verdadera situación empresarial queda oculta hasta que la empresa llega a la quiebra.

Las prácticas de buen gobierno son la respuesta de las empresas para volver a ganarse la confianza del mercado, estas prácticas van encaminadas a impulsar a las empresas a que suministren información transparente sobre aspectos organizativos, estratégicos y sobre el contenido en sí de la información financiera que se divulgue. Las prácticas de buen gobierno han desembocado, por una parte, en la necesidad que las empresas publiquen informes de gobierno, y por otra, que los gobiernos de los distintos países hayan promulgado los conocidos Códigos de Buen Gobierno.

Los Códigos de Buen Gobierno, pretenden dirigir la estructura del gobierno de las empresas y guiar la actuación de las mismas. De esta forma, el Gobierno Corporativo es un conjunto de reglas que guían y limitan la acción de los gerentes. Los Códigos de Buen Gobierno vienen a establecer los fundamentos de la Gobernanza para las empresas.

Autores como Milton Friedman (1970) o lo impulsores de la Teoría de la Agencia Jensen y Mecking (1976) tenían una visión de la empresa basada únicamente en los accionistas y el objetivo de la misma se centraba en maximizar el beneficio y el valor para los accionistas. La teoría de los "stakeholders" amplía el concepto, planteando que las empresas deberán rendir cuentas no sólo ante sus accionistas sino ante un grupo más amplio de partes interesadas entre los que se encuentran, los acreedores, accionistas, trabajadores, clientes, sindicatos, la comunidad local y la sociedad en general. Pero además se encuentran los inversores institucionales, derivado del creciente aumento de los planes de pensiones que canalizan el ahorro a través de los mercados financieros.

Todos ellos son los llamados "stakeholders". De la satisfacción de sus demandas depende la supervivencia de la empresa. Se puede pensar que todos los partícipes a largo plazo tienen los mismos intereses (supervivencia y creación de valor), sin embargo, a corto plazo no es así, ya que los diferentes "stakeholders" tienen intereses y necesidades informativas diferentes, estas diferencias de intereses influyen en los objetivos y en las decisiones empresariales, además las empresas deben identificar, jerarquizar a los "stakeholders" y buscar estrategias que no 
perjudiquen a ningún grupo. En este sentido la gestión de los "stakeholders" es crucial para la empresa.

Las empresas deben cambiar su mentalidad y considerar en la definición de sus estrategias no solo aspectos económicos sino también éticos (Bonnafous-Boucher 2005), esto supone abandonar la teoría, y tener objetivos más allá, de la maximización del beneficio. El resultado económico, no es el único parámetro a ser medido. Es necesario ampliarlo hacia el desempeño empresarial y buscar indicadores que muestren como se satisfacen las necesidades de los distintos "stakeholders". En definitiva, todo va a girar en torno a las demandas de los "stakeholders", las cuales pueden tener repercusiones en los resultados empresariales. En opinión de Hart y Milstein, (2003), a corto plazo, un gobierno bien estructurado puede adoptar políticas que conducen a una asignación de recursos más eficientes cuando se amplia el horizonte temporal hasta el largo plazo, favoreciendo con ello el crecimiento económico. Las prácticas de buen gobierno tratan de solucionar los problemas que pueden plantearse entre los directivos y los "stakeholders" estableciendo un sistema de control interno y externo de la conducta empresarial. Las prácticas de buen gobierno podrían poner de manifiesto recursos estratégicos que puedan ser capaces de generar ventajas competitivas que tengan repercusión en la rentabilidad.

La preocupación por el buen gobierno de las empresas y la defensa de los intereses de los "stakeholders", ha conducido a que en los últimos tiempos todos lo países desarrollados hayan promulgado diferentes Códigos de Buen Gobierno. Sin embargo, el grado de desarrollo de los mismos no ha sido igual, ya que, indistintamente de la fecha de promulgación de los distintos códigos, la mayoría de las empresas de los países de la Europa Continental empezaron a emplear prácticas de buen gobierno a principios del presente siglo, mientras que las empresas del área de influencia anglosajona llevan haciéndolo desde la década anterior. Por tanto, el desarrollo y la influencia que estas prácticas han tenido en las empresas son diferentes dependiendo de cada país. Y ello, es consecuencia de la distinta importancia que ha tenido el mercado en la financiación de las empresas. 


\section{BUENAS PRÁCTICAS CORPORATIVAS \\ 3. LA DIVERSIDAD COMO FACTOR RELEVANTE PARA LAS}

Uno de los parámetros mediante los que se miden las prácticas de buen gobierno es la información que emiten las empresas sobre su consejo de administración (composición, retribución...), y dentro de ello, se le concede importancia a la diversidad como factor relevante para las buenas prácticas corporativas.

Aunque, como veremos a continuación, no se puede afirmar de una forma contundente la relación positiva entre diversidad y rendimiento empresarial, si se puede defender la bondad de la diversidad para el buen gobierno de la empresa (Farrel y Hirsch 2005).

En un principio pueden distinguirse dos tipos de diversidad: diversidad "observable" (edad, género, raza...) y otra diversidad que puede ser clasificada como "no observable" (conocimiento, educación, estudio, personalidad...). La dificultad de cuantificar la diversidad "no observable" hace que se hable siempre de diversidad "observable", que puede ser definida como la presencia en los consejos de administración o en los grupos de dirección de personas de distinto: género, raza, religión...

Tradicionalmente los consejos de administración han sido y siguen siendo, en muchos casos, grupos homogéneos, formados principalmente por hombres con perfiles similares en cuanto a edad, profesión, educación etc., e incluso con la misma visión de los negocios. Aunque, esta situación puede ser un poco diferente cuando hablamos de consejeros dominicales.

La sociedad en la que se encuentran ubicadas las empresas se caracteriza por su falta de homogeneidad. Hoy en día las empresas actúan en economías complejas y la gestión de las mismas requiere de personas poseedoras de cualidades, habilidades y capacidades necesarias para gestionar adecuadamente en entornos complejos.

Si los consejos de administración son los órganos de gobierno de una empresa, la cual actúa en un entorno complejo no parece descabellado pensar que la introducción de la diversidad en los mismos hará que esté mejor representada la realidad. Además de este factor y de otros que veremos posteriormente, las voces a favor de la diversidad en los consejos de administración pueden venir por el intento que están haciendo todas las instancias institucionales para desembarcar la preponderancia masculina en todos los niveles de la sociedad. Esta 
situación ha llegado de pleno a las organizaciones públicas (política de paridad) pero no se puede decir lo mismo en las empresas privadas. Por este motivo los distintos gobiernos se han preocupado, en mayor o menor medida, de introducir en los Códigos de Buen Gobierno temas relativos a la diversidad en los consejos de administración. Además la cuestión, no es sólo diversidad si no como se gestiona la diversidad. Con frecuencia los efectos positivos de consejos de administración diversos no se manifiestan si los prejuicios impiden que se lleve a cabo una adecuada gestión de la diversidad.

La diversidad y su gestión están de actualidad especialmente en relación con la participación de las mujeres en las cúpulas directivas de las empresas y organizaciones, demandada por organizaciones feministas, partidos políticos y movimientos sociales de diversos tipos.

Aunque se justifique por si sólo, el querer terminar con una discriminación que existe desde hace mucho tiempo, el incorporar consejeras independientes a los consejos de administración puede deberse también a razones puramente competitivas y de negocio. En definitiva, las razones por las que se considera esencial evitar los sesgos y prejuicios de género, raza u otros, que pueden afectar negativamente a los resultados y la eficacia de las organizaciones son (Robinson y Dechant 1997):

- Ahorro de costes y aprovechamiento del talento interno (tasas de rotación laboral más bajas, lo que favorece la curva de aprendizaje; tasas de absentismo y de litigación más bajas).

- Atracción, retención y promoción del talento externo (si se admite la diversidad y se gestiona adecuadamente).

- Crecimiento del negocio derivado de la mejor comprensión de los mercados (consumidores y proveedores); mayor creatividad e innovación; calidad en la resolución de problemas; refuerzo efectivo del liderazgo y creación de relaciones globales.

Burgess y Tharenou (2002) insisten por su parte en que la principal ventaja de los grupos heterogéneos frente a los de composición más homogénea es que éstos últimos tienden a resolver todos los problemas de la misma manera y, por tanto, a repetir errores de apreciación, algo menos probable en grupos diversos. En relación con la presencia de mujeres en los consejos de administración, concretan las razones a favor de la diversidad en lo siguiente: más contraste de opiniones; nueva información estratégica para el consejo, puesto que las mujeres deciden un 
porcentaje muy elevado de las compras de los hogares; influencia en el estilo de liderazgo y toma de decisiones de la organización; garantía de un mejor comportamiento de los consejos de administración y del gobierno; female role model para el gran número de mujeres preparadas y dispuestas a participar en consejos de administración.

\section{EMPRESAS}

\section{LA DIVERSIDAD DE GENERO FACTOR CLAVE PARA LAS}

La incorporación de las mujeres al mercado de trabajo ha sido paulatina pero sin pausa, y en la actualidad las mujeres ocupan cargos que hasta el momento habían sido reservados para los hombres. Las mujeres han alcanzado niveles de formación y cualificación muy elevados, pero aún el hecho de pertenecer a uno u otro sexo sigue condicionando las posibilidades que tienen las personas de acceder a determinados puestos.

La discriminación a la que están sometidas muchas mujeres, motivada por una gran cantidad de factores invisibles provocan no sólo situaciones injustas, sino también pérdidas de talento para las organizaciones que lo practican. Esta situación ha hecho que la comunidad científica de las disciplinas sociales se preocupe por estas cuestiones relacionadas con la política de igualdad de oportunidades, sin embargo en la literatura del ámbito económico-empresarial este problema parece tener menor eco.

Como acabamos de señalar las ciencias sociales se han ocupado de este problema; en este sentido existen trabajos como el de Barberá (2000), en el que se rebaten los argumentos acerca de que las mujeres tienen menor nivel formativo que los hombres y muchos que tratan el denominado "techo de cristal"1, como el de Alimo-Metcalfe (1995) en el que se demuestra la existencia de promociones en la alta dirección de las empresas de carácter no formal, el de Ragins et al. (1998) en el que se pone de manifiesto la falta de un desarrollo de una carrera adecuada para las mujeres. Pero entre todos ellos destaca el de

\footnotetext{
La expresión "techo de cristal" fue acuñada hace veinte años por el departamento de trabajo del gobierno federal estadounidense para referirse a "aquellas barreras artificiales basadas en prejuicios actitudinales u organizativos que previenen el avance vertical en las organizaciones de individuos cualificados" (Powell y Butterfield 1994).
} 
Wirth (2002) "Romper el techo de cristal: las mujeres en puestos de dirección", publicado por la OIT (Organización Internacional del Trabajo), en el que se han tomado como base un conjunto de grandes empresas pertenecientes a los países con mayores niveles de desarrollo económico y que arroja conclusiones similares al resto de los trabajos referenciados.

El área de Recursos Humanos también se ha ocupado de este problema, abordándolo desde la perspectiva de la diversidad, de este modo, trabajos como el de Kandola y Fullerton (1998) o el de Rosenzweig (1998) aportan explicaciones sobre como la gran complejidad y dinamismo que caracteriza al entorno, demanda organizaciones constituidas por personas, cada vez más diversas, que les permitan encajar en la nueva cultura empresarial. Otro tipo de trabajos como los de Benschop (2001) y McDouglas (1996), dedicados a la gestión de la diversidad, proponen modelos para explicar como su implantación puede sentar las bases hacia la obtención de desempeños organizativos superiores. Burgess y Tharenou (2002) y Pounder y Coleman (2002), señalan que si las empresas quieren adaptarse al nuevo contexto económico, deberán utilizar nuevos estilos de liderazgo y formas de organización, pues el mercado laboral demanda estilos de dirección que incorporen características tradicionalmente femeninas. El desarrollo de las empresas dependerá de la capacidad de su dirección para gestionar su recurso más valioso: las personas.

En los anteriores trabajos la diversidad tiene una gran importancia en las organizaciones, y es considerada como un elemento enriquecedor de innovación y adaptación a las necesidades actuales. La igualdad entre los géneros potencia la productividad y estimula el crecimiento económico. La igualdad entre sexos aprovechará al máximo los recursos humanos disponibles en las empresas, así como las posibles ventajas que puede tener la existencia de participación femenina en los equipos de dirección, y además comprobar como la unión de las diferencias entre hombres y mujeres puede generar más poder que el que se pueda alcanzar por separado.

Por último, existe un grupo de trabajos que, desde el punto de vista de la diversidad, argumentan que la presencia de mujeres en los puestos de toma de decisiones de las compañías repercute de una forma positiva en el éxito empresarial. Robinson y Dechant (1997) argumentan que las empresas en las que su cúpula directiva está formada por hombres y mujeres, los cuales aportaran diferentes habilidades, conocimientos y experiencias, promoverán más y mejor la creatividad y la innovación empresarial. El informe Tyson (2003) concluye que la diversidad en los 
consejos de administración llevará a un mejor funcionamiento de la compañía, debido fundamentalmente a la existencia de diferentes puntos de vista.

Siguiendo con la argumentación de la diversidad, los trabajos de Adler (2001), Carter et al. (2003) y el informe Catalyst (2004), así como el de Jimeno y Redondo (2005) para España, encuentran una relación positiva entre la presencia de mujeres en los puestos de toma de decisiones empresariales con el valor de la empresa, medido este valor por diferentes indicadores como la rentabilidad económica o la rentabilidad financiera. En contra de los anteriores trabajos están los de Monacci (1997) o el de Ohlott et al. (1994) en los que no se encuentra relación entre la rentabilidad empresarial y la presencia femenina en la dirección de la compañía.

Todo lo anterior, pone de manifiesto que no existe una relación clara entre rentabilidad y género, aunque si se pueda defender la bondad de la diversidad para el buen gobierno de la empresa. Será por tanto la diversidad el argumento para justificar la presencia de mujeres en los puestos de toma de decisiones empresariales (Farrel y Hirsch 2005).

Todos estos trabajos desmontan en cierta medida los tópicos respecto a la existencia de hipótesis acerca de la falta de habilidades directivas por cuestiones de género. Ya se está produciendo en algunas organizaciones, al menos en las más vanguardistas, un cambio de paradigma, en el que se predica que con lo que cuenta una organización es con su personal y el talento y formación del mismo. Esto sin duda influirá a la hora de crear el ambiente necesario para obtener las condiciones que favorezcan el desarrollo de las potencialidades, independientemente del sexo. El talento se encuentra tanto en los hombres como en las mujeres, y es una pérdida importante para las empresas su desaprovechamiento.

\section{EUROPEOS}

\section{LA DIVERSIDAD EN LOS CÓDIGOS DE BUEN GOBIERNO}

En Europa, desde principios de los 90 se han publicado numerosos códigos de buen gobierno en casi todos los países europeos. La gran mayoría se han diseñado o revisado en los tres últimos años con el objeto de recoger en ellos las nuevas normas recomendadas por los organismos reguladores de los mercados de valores de cada país y por la situación real del entorno empresarial, y han tomado como base tanto las recomendaciones de la Unión Europea como la de la OCDE. 
La OCDE emitió unos principios sobre gobierno corporativo en 1999. En 2004, se revisaron estos principios para incorporar los avances que se habían producido tanto en los países miembros, como en los no miembros en esta materia. Esta revisión se sigue basando en un planteamiento no vinculante y de principios, que reconoce la necesidad de adaptar su implantación a las circunstancias cambiantes en el entorno legal, económico y cultural de cada país.

La globalización de la economía y la necesidad de la Unión Europea de contar con un mercado de capitales transparente que cuente con sistemas de gobierno corporativo claros y preocupados por todas las partes interesadas ("stakeholders"), planteó la posibilidad de elaborar un código unificado que se recogiesen las pautas por las que se deberían regir las empresas. Pero con la emisión en Europa del Informe Winter en noviembre de 2002 (UE 2002), se descartó la idea de un código europeo único de buenas prácticas y se abordó una serie de cuestiones esenciales en materia de gobierno corporativo sobre transparencia informativa; derechos de los accionistas; comité de auditoría, y sobre las comisiones de nombramientos y remuneración. Adicionalmente, como desarrollo de este informe se emitió por la Comisión Europea recomendaciones sobre remuneración y sobre los consejeros independientes y las comisiones delegadas (CE, 913/2004/CE; CE, 162/2005/CE). Estas recomendaciones han influido en los códigos emitidos en los distintos países. La Unión Europea ha recomendado que sus estados miembros establezcan sus propios códigos de prácticas teniendo en cuenta sus características nacionales específicas y reflejando la diversidad de sistemas y prácticas de gobierno corporativo en la Unión Europea.

Por tanto, a pesar de las diferentes culturas, tradiciones financieras, estructuras organizativas y orígenes legales de los diferentes países, así como la forma en que las empresas están intentando competir en el mercado global, los códigos de gobiernos elaborados en casi todos los países europeos presentan similitudes entre ellos, pero con características propias $^{2}$.

El primer informe sobre gobierno corporativo en Europa fue el Informe Cadbury en Reino Unido 1992. Desde esta fecha hasta la actualidad se ha ido perfeccionando el tema emitiendo diferentes documentos, como

2 Todos los CBG están publicados en la página http://www.ecgi.org/ 
las recomendaciones de los consejeros de Greenbury (1995), el informe Higgs (2003) y el Informe Smith (2003). Con estos documentos se va actualizando el código, el Código Combinado de Gobierno Corporativo de Junio de 2006 es la última versión publicada hasta ahora.

Francia fue el segundo país europeo en tratar el tema. Los principios para el gobierno corporativo de las sociedades cotizadas en este país están basados en los Informes Viénot de Julio de 1995 y Julio de 1999, y en el informe Bouton de Septiembre de 2002. En Octubre de 2003, se emite un documento que aglutina los tres, agrupando y ordenando las recomendaciones y eliminado aquéllas que ya estaban obsoletas.

A partir de 1997 empiezan a publicarse documentos relacionados con el tema en casi todos los países Europeos. Holanda, con el informe Peter, que posteriormente ha sido objeto de revisión y sustituido por el Código de Diciembre de 2003. En Alemania se emiten diversos documentos sobre el tema en 1998, el Informe Baums se publica en julio de 2001 y en 2002 se publica el Código Cromme cuya última versión es de junio de 2006.

España también elabora el Informe Olivencia en 1998 y el Aldama en 2003, y en 2006 emite el Código Unificado, estos códigos son objeto de comentarios más adelante.

Portugal emitió en 1999 recomendaciones sobre gobierno corporativo, que han sido objeto de revisión en 2003 y 2006. Italia, también emitió en Octubre de 1999 el código Preda revisado en julio 2002, y en Marzo de 2006.

En Dinamarca se elaboró una pequeña guía en el 2000 y en Diciembre de 2001 se publica el Informe del Comité Nǿrby y en 2002. Posteriormente se han revisado en 2003 y 2005. El Código Suizo se emitió en junio de 2002, sobre la base de los códigos anteriormente publicados en Inglaterra, Alemania y Francia. Finlandia, en Diciembre de 2003 para las sociedades cotizadas y en Enero de 2006 para las no cotizadas.

Islandia publicó en Marzo de 2004 una Guía aplicable a todo tipo de sociedades cotizadas o no. Noruega publicó en diciembre de 2004 el Código que ha sido objeto de revisión en 2005 y 2006 para introducir los cambios producidos en la regulación noruega. En Suecia, desde 1993 se han ido emitiendo por distintas asociaciones y comités normas sobre el tema. En abril de 2003, se forma un grupo de trabajo conjunto para emitir un código de gobierno corporativo único aplicable a las empresas suecas y en Diciembre de 2004 se publicó el informe definitivo. 
Hasta 2004 Bélgica tenía tres documentos que contenían recomendaciones sobre gobierno corporativo de las sociedades cotizadas, emitidos en 1998. En enero de 2004 se formó un comité único que emitió en el mismo año un Código. En Austria se emiten documentos relacionados con el tema en 2002 y 2006, Luxemburgo ha sido el último país de los quince en emitir recomendaciones sobre el tema (2006). Incluso los países incorporados en los últimos años a la Unión Europea han emitido Códigos de Buen Gobierno. El único país de la Unión Europea que no tiene ningún documento sobre el tema es Bulgaria.

Cada código presenta una estructura diferente, pero todos abarcan una serie de relaciones entre el cuerpo directivo de una empresa, su consejo de administración, sus accionistas y otras partes interesadas. El papel de cada uno de estos participantes y la interacción entre los mismos varían enormemente en función del país. En el Estudio Comparativo de los Códigos de Gobiernos realizado en la Unión Europea en 2002 (Gregory 2002), se puso de manifiesto que las mayores diferencias en las prácticas de gobierno corporativo entre países derivan de las distintas normativas propias de cada uno de los países, más que de las diferencias en las recomendaciones de gobierno corporativo. Por ejemplo, en Alemania y Noruega los empleados tienen un papel significativo dentro de la organización, bien sea participando en el Consejo de Administración o formando parte de una asamblea corporativa específica.

En casi todos los países se han ido incorporando parte de las recomendaciones dentro de la normativa de obligado cumplimiento. Esto ha determinado que en algunos países, como Noruega, se distingue entre los términos debe y debería para expresar esa diferencia. En otros, se hace referencia expresa a lo que supone una obligación legal para diferenciarlo de la simple recomendación.

Uno de los puntos que trata los Códigos de Buen Gobierno, para asegurar que el consejo de administración pueda operar de forma independiente a intereses particulares y realice un control eficiente sobre el equipo de gestión, es la composición del consejo. En esta composición se establece la necesidad que sean diversos los componentes del consejo tratando de salvaguardar los intereses de todos los accionistas y no solo los intereses de los accionistas de control o mayoritarios. En todos los Códigos se recomienda que el consejo de administración esté formado por consejeros ejecutivos y no ejecutivos (externos). Igualmente se recomienda que la función de presidente del consejo y el primer ejecutivo no recaiga sobre la misma persona. Sin embargo, varía la intensidad de las 
recomendaciones en cada Código y la práctica de cada país. En Noruega, normalmente, no se eligen representantes del equipo ejecutivo para el consejo de administración a pesar de que el código Noruego recomienda que al menos la mitad de los miembros del consejo sean externos, pero añade que ni el primer ejecutivo ni ningún otro miembro del equipo ejecutivo deberían ser miembros del consejo. En Inglaterra, sin especificar proporciones, se establece como principio fundamental que el consejo esté formado de forma equilibrada por consejeros ejecutivos y no ejecutivos de tal forma que ningún individuo o pequeño grupo pueda dominar la toma de decisiones del consejo. En Italia el número de consejeros externos sobrepasa normalmente al de los ejecutivos, y el código recomienda que cada empresa debería determinar el número de los externos en relación a su tamaño y del total de miembros del consejo, pero que debería ser tal que puedan llevar el peso significativo en la toma de decisiones. Tanto el Código Italiano como otros Códigos europeos recomiendan que en caso de que el presidente del consejo y el primer ejecutivo recaiga sobre la misma persona se debería nombrar un consejero independiente coordinador.

Igualmente todos los Códigos recomiendan que una parte de los consejeros externos sean independientes, para representar los intereses minoritarios de los accionistas y evitar que uno o más accionistas significativos (consejeros externos dominicales) puedan en la práctica manipular las decisiones del consejo. También coinciden en las características generales que deben tener un consejero para considerarse como tal. Aunque, la descripción de estas condiciones es más detallada conforme se perfeccionan los códigos.

Referente a este punto, Reino Unido establece que al menos la mitad del consejo excluyendo el presidente, deberían ser consejeros externos independientes y las pequeñas empresas deberían tener al menos dos consejeros independientes. Recomienda también que se elija un consejero independiente coordinador para facilitar el contacto con los accionistas. El Código Noruego, recomienda que al menos dos de los miembros del consejo debería ser independiente y, al igual que el Danés, que de los consejeros elegidos por los accionistas al menos la mitad deberían ser independiente. El Código Alemán sólo recomienda que en el consejo de administración debería haber un número suficiente de miembros independientes, considerando independiente cuando no existe ningún tipo de relación comercial o personal con la sociedad o su junta directiva. El Código Italiano tampoco considera necesario emitir una cantidad determinada, señalando únicamente que un adecuado número de consejeros 
externos deberían ser independientes. El mismo Código Italiano comenta que los consejeros externos enriquecen las discusiones del consejo con la experiencia propia, ofreciendo puntos de vistas diferentes que contribuyen a la toma de decisiones más adecuadas. Además señala que estos consejeros son muy importantes en asuntos donde los intereses de los consejeros ejecutivos y los intereses de los accionistas pueden no coincidir.

Dentro de la composición del consejo de administración, los Códigos hacen referencia, expresa o implícitamente a la diversidad como buena práctica de gobierno. El Código Noruego recomienda que "la composición del consejo debería realizarse teniendo en cuenta que los consejeros puedan atender a los intereses comunes de todos los accionistas y reunir la necesaria experiencia, capacidad y diversidad de la sociedad" . $^{2}$ En los comentarios a este punto, señala que además de tener la apropiada experiencia, es importante que el consejo tenga suficiente capacidad para llevar a cabo sus deberes. Esto significa, en la práctica, que cada miembro del consejo debe tener tiempo disponible suficiente para dedicarse a su puesto como consejero. No se limita las actividades fuera del consejo, pero se llama la atención a los conflictos de intereses que pueden surgir si tienen otros compromisos. Adicionalmente se recomienda que para que el trabajo que ha de desarrollar el consejo se realice de forma adecuada, debe existir una amplia diversidad en su composición. En este aspecto señala: "se debería poner especial atención en establecer un equilibrio entre miembros hombres y miembros mujeres en el consejo; que estén representado todos los grupos de accionistas y se potencia también la capacidad de trabajar en equipo".

Atendiendo a estas recomendaciones el gobierno Noruego ha realizado una apuesta fuerte a favor de la diversidad de género en los consejos, ya desde Enero de 2004, las empresas públicas tienen que tener una representación de cada sexo de al menos el $40 \%$ del consejo de administración, en cuanto al sector privado, ha tenido un plazo de adaptación hasta julio de 2005, con penalizaciones previstas para 2007 en caso de que las empresas no cumplan con lo previsto. A marzo de 2004, el promedio de mujeres que formaban parte de los consejos era del $22 \%$, el mayor porcentaje de todos los países europeos.

3 La cursiva es nuestra. 
Ni el Código Italiano ni el Alemán hacen referencia expresa a la diversidad, aunque señalan que han de poseer conocimientos, capacidad y experiencia profesional suficiente. Otros Códigos, como el Danés añaden la necesidad de experiencia y conocimientos internacionales.

El Código de Reino Unido establece que debe existir un equilibrio adecuado entre conocimientos y experiencias necesarias para el desarrollo de las funciones del consejo. Que el proceso de selección de nuevos consejeros debe ser formal, riguroso y transparente, y que se debe realizar de tal forma que los elegidos tengan tiempo disponible suficiente para desarrollar sus tareas. El Informe Higgs (2003), pone de relieve que los consejos deberían seleccionar un número mayor de consejeros no ejecutivos, a ser posible con mayor participación femenina, así, se acabarían constituyendo consejos de administración más diversos, caracterizados por ser más efectivos y activos. Esta misma visión la comparte el Informe Tyson (2003), ratifica que aquellos consejos de administración que integren la idea de diversidad en su funcionamiento, ya sea de género, religión, nacionalidad, se caracterizan por un mejor funcionamiento. Por un lado, son el reflejo de la diversidad también presente en los grupos de interés concurrentes, por otro, continúa, los consejos de administración diversos constituyen una base de conocimiento, aptitudes y experiencias que permite enfrentarse a problemas desde distintos puntos de vista; así se trataría de evitar la unidireccionalidad del pensamiento en los consejos de administración.

El código Finlandés también señala que la edad media y la proporción entre ambos sexos pueden ser consideradas en la composición del consejo. Igualmente el Código Sueco recoge que debería conseguir igualdad de género en su composición.

\section{LA DIVERSIDAD EN EL CÓDIGO UNIFICADO ESPAÑOL}

En España en 1997, el Gobierno encomendó a una comisión presidida por el profesor Olivencia la elaboración de un Código ético dirigido a las sociedades cotizadas. En febrero de 1998 se publica el Código de Buen Gobierno por la Comisión Olivencia. Su aplicación era completamente voluntaria, y se recomendaba a las empresas publicar información sobre sus reglas de gobierno, razonando las que no se ajustaran a las recomendaciones del código. Se esperaba que los mercados, a los que llegara esta información, recompensaran las prácticas de buen gobierno y sancionaran negativamente su incumplimiento. 
Teniendo en consideración el Informe Olivencia, las experiencias recogidas de su escasa aplicación y los retos adicionales que habían surgido desde que se aprobó dicho informe, se publicó en el año 2003 el Informe, rendido por la Comisión Aldama, para el fomento de la transparencia y seguridad en los mercados y en las sociedades cotizadas. Su recomendación esencial, hacer obligatoria la difusión por las sociedades de más información sobre su régimen de gobierno, tuvo plasmación casi inmediata en la Ley de Transparencia del mismo año. De esa y de otras normas que la desarrollaron surgió un exigente régimen de información sobre la materia de gobierno corporativo que, en opinión del presidente de la CNMV no tiene parangón ni en el resto de la Unión Europea ni en otros países miembros de la IOSCO (CNMV 2005).

Adicionalmente, la actualización de los principios de gobierno corporativo de la OCDE (2004), el impulso que la Comisión Europea ha venido dando al fomento del buen gobierno de las sociedades cotizadas europeas, llevó al gobierno español, en Julio de 2005, a solicitar de un grupo de expertos dirigidos por el presidente de la CNMV, la puesta al día del Código de recomendaciones sobre buen gobierno.

El 18 de enero de 2006 se publicó el Proyecto de Código Unificado que se sometió a consulta pública y después de numerosas alegaciones se aprobó definitivamente en Mayo de 2006. El Código además de refundir las recomendaciones formuladas por las Comisiones Olivencia y Aldama, ha tomado como guía las recomendaciones de la Comunidad Europea y las recomendaciones de buen gobierno de las entidades de créditos aprobadas por el comité de supervisores bancarios de Basilea, actualizando la materia en determinados puntos. El Código se centra, únicamente, en aquellas recomendaciones que no se recogen en normativas obligatorias.

El proyecto de Código Unificado fue duramente criticado. El siguiente artículo de prensa es buen ejemplo de ello:

La presentación del proyecto Código de Buen Gobierno, ... ha caído como un jarro de agua fría entre las empresas cotizadas. A pesar de su carácter voluntario, el texto utiliza un tono imperativo que ha asustado a unas compañías, que están muy lejos de cumplir las nuevas recomendaciones sobre consejeros independientes, presencia de mujeres en los consejos, transparencia en las retribuciones, funcionamiento de las comisiones... (El País 22 de enero 2006). 
Las críticas y comentarios al proyecto dieron lugar a algunos cambios: unos de forma, y otros de fondo. Así se pasó a utilizar el subjuntivo y no el futuro para evitar el tono imperativo y se redujo el número de recomendaciones; se suprimió la figura del vicepresidente como contrapeso al presidente ejecutivo, utilizándose la figura del consejero independiente coordinador, se suprimió el requisito que los secretarios no sean consejeros, y se aceptó que puede ser independiente el que posea un paquete inferior al $5 \%$.

Pero se han mantenido cuestiones sustanciales a pesar de haber sido objeto de numerosas polémicas. Entre ellas destacamos las siguientes:

* El Código se basa en el principio de voluntariedad de cumplir y explicar, ya recogido en los anteriores códigos, en la Ley de mercado de Valores y en línea con las directivas Europeas y de la OCDE, permitiendo libertad a las empresas de autogobernarse y dejando al mercado que decida si su gestión es aceptable.

* El Código representa una defensa de los Consejeros Independientes, definiendo específicamente las condiciones mínimas para ser considerado como tal, y que constituyan al menos $1 / 3$ del Consejo.

* Recomienda que el número de Consejeros Ejecutivos sea el mínimo necesario. El código no se pronuncia sobre la separación entre el cargo de Presidente del Consejo y del Ejecutivo, ya que no hay uniformidad en la práctica internacional ni evidencia empírica de si es mejor, pero si establece la evaluación del Presidente y la figura del Consejero Independiente coordinador como contrapeso.

* Enfatiza el papel primordial de la Comisión de Nombramientos en la defensa de la independencia y de perseguir el interés social por todos los miembros del Consejo.

* Recomienda que el informe de retribuciones sea objeto de voto consultivo por la Junta General y que debería incluir retribuciones de todos y cada uno de los consejeros y por cualquier concepto.

* Incorpora novedades en la Independencia de la Auditoría y recomienda que los estatutos no contengan limitaciones del derecho de voto ni otras medidas anti-OPA, así como que las sociedades establezcan mecanismo para poder denunciar confidencialmente las irregularidades observadas en la sociedad (financiero contable).

* Y por último, reconoce la necesidad de aumentar la participación de la mujer en los consejos de administración, para lo cual recomienda 
que cuando sea escaso o nulo el número de consejeras, el consejo deberá explicar los motivos y las iniciativas adoptadas para corregir tal situación. En particular, la comisión de nombramientos debería velar para que al proveerse nuevas vacantes: los procedimientos de selección no adolezcan de sesgos implícitos que obstaculicen la selección de consejeras; la compañía busque deliberadamente, e incluya entre los potenciales candidatos, mujeres que reúnan el perfil profesional buscado.

El Código Unificado, a pesar de la polémica suscitada, dedica el apartado 15 a la diversidad, y como punto novedoso respecto a los informes Olivencia y Aldama, recomienda que el consejo refleje la diversidad de conocimientos, de género y de experiencias precisas para desempeñar sus funciones con eficacia, objetividad e independencia.

En concreto, respecto a la diversidad de género, explica que estas recomendaciones parten de la convicción que lograr una adecuada diversidad de género no es una mera cuestión ético-política o de "responsabilidad social corporativa" sino también un objetivo de eficiencia, al menos a medio plazo: desaprovechar el potencial talento empresarial del $51 \%$ de la población -las mujeres- no puede ser económicamente racional en el conjunto de las grandes empresas de nuestro país. Las recomendaciones parten igualmente de que el predominio de hombres en puestos de responsabilidad es un fenómeno en parte auto-inducido, esto es, el predominio de hombres, hace más probable que se sigan nombrando consejeros de ese sexo, de forma que la falta de diversidad no se corregirá sin un esfuerzo deliberado para lograrlo (CNMV 2006a). El Código invita a las sociedades con escasa presencia femenina en sus Consejos a que hagan un esfuerzo deliberado por buscar posibles candidatas cada vez que deba cubrirse alguna vacante en el Consejo, especialmente para puestos de independientes (CNMV 2006b).

La Comisión de Nombramientos y Remuneración (generalmente combinada en una misma) cobra una gran importancia como protagonista en los procesos de selección y nombramientos de nuevos Consejeros verdaderamente Independientes y procurando activamente la entrada de más mujeres. 


\section{LA DIVERSIDAD EN LA PRÁCTICA EMPRESARIAL}

Como destaca Llopis (2006), desde que el Código Olivencia se publicó (1998) hasta ahora, ha habido grandes cambios: la Comisión de Auditoría como tal pasó de existir en el 73\% de las empresas del IBEX a estar en la totalidad de ellas. Otro cambio importante fue el de los Consejeros Independientes que pasaron del 21\% al 36\%, aunque cada empresa definía independiente a su manera, sin criterios claros y consistentes. Los cambios menores y menos perceptibles han sido la reducción de presidentes ejecutivos del 68\% al 64\% y el aumento de consejeras mujeres (excluyendo las dominicales o familiares) pasando de 12 puestos en el IBEX 35 a 44 en el 2005.

El estudio de Heidrick-Struggles $(2005)^{4}$ muestra un progreso significativo en las prácticas de buen gobierno de diez los países europeos objeto de su estudio desde 1999, además de existir menores diferencias entre países. Las mejores calificadas han sido Inglaterra (1 $\left.1^{\mathrm{a}}\right)$ Holanda $\left(3^{\mathrm{a}}\right)$ y Francia $\left(4^{\circ}\right)$, pasando Suiza en 2005 a ocupar la segunda posición. Suecia ocupa la $5^{a}$ posición. España y Portugal son las que han tenido cambios más significativos pasando de las últimas posiciones a la $6^{\mathrm{a}}$ y $8^{\mathrm{a}}$ posición respectivamente. Bélgica ha pasado de la quinta a la séptima posición y las peor calificadas han sido Alemania $\left(9^{\mathrm{a}}\right)$ e Italia $\left(10^{\mathrm{a}}\right)$.

No obstante, las cifras todavía no son muy alentadoras. El Informe de Gobierno Corporativo de las entidades emisoras de valores de 2004 (CNMV 2005), expone, que en el 33,7\% de las sociedades cotizadas existe un accionista de control. En relación a la diversidad de género señala que el promedio de mujeres en los consejos se sitúa en el 5,9\% de sus miembros, mientras que en las compañías del IBEX desciende hasta el $3,4 \%$. El $60 \%$ de las sociedades cotizadas no cuentan con la presencia de mujeres en sus consejos de administración y en el caso de las compañías del IBEX ese porcentaje se eleva hasta casi el $70 \%$. Y, que la mayoría de las mujeres tienen la condición de dominicales $(57,7 \%)$ al haber sido nombradas en representación de paquetes accionariales significativos. Las ejecutivas representan tan solo el $19,2 \%$.

4 Este estudio se ha realizado sobre diez países europeos Reino Unido, Suiza, Noruega, Francia, Suecia, España, Bélgica, Portugal, Alemania e Italia, citados en el orden de ranking asignado en 2005. 
El estudio elaborado sobre diversidad por la Fundación de Estudios Financieros (Gómez 2005) pone de manifiesto que los consejos de administración españoles se encuentran en las últimas posiciones, en cuanto a diversidad de género, de entre los países europeos. En este mismo estudio hace referencia al Informe EPWN (2004), que sitúa a España, junto con Bélgica e Italia entre los países europeos con una menor presencia de mujeres en los consejos. Italia con un $2 \%$ es el país que ocupa la última posición del ranking. De las 15 sociedades italianas que forman parte de la muestra, únicamente 2 tienen presencia femenina en los consejos. En el polo opuesto se encuentran los países nórdicos con Noruega a la cabeza con un $22 \%$, las suecas con un $20 \%$ y las finlandesas con un $14 \%$. Reino Unido, con un $10 \%$ de mujeres consejero frente al $5 \%$ en el 2000 , Alemania con otro $11 \%^{5}$, y Francia con un $7 \%$, ocupan las posiciones intermedias de diez países que muestra el informe elaborado por Heidrick-Struggles (2005). Portugal tiene la más baja proporción de mujeres en los consejos: un $1 \%$. Suiza a pesar de haber conseguido el mayor grado de mejora en el ranking de gobierno corporativo la proporción de consejeros mujeres está por debajo de la media europea $6 \%$.

Este estudio de Heidrick-Struggles (2005), señala que aunque sus estadísticas muestran un $22 \%$ de aumento en el número medio de mujeres en los consejos europeos desde 2003 a 2005. Esta tasa es de una muy baja base (subió de $6 \%$ en 2003 a 7,3\% en 2005). 46\% de las empresas europeas estudiadas todavía no tienen mujeres en sus consejos (contra 54\% hace dos años). Y continua señalando que la baja incidencia de consejeros femeninas esta claramente vinculado con la falta generalizada de mujeres en los más altos puestos de los negocios europeos.

También se ha puesto de manifiesto que la importancia femenina en los consejos también está asociada al porcentaje de sociedades que cuentan con al menos una mujer entre sus consejeros. El promedio europeo es del 62\% (EPWN 2004) con países como Noruega, Suecia, Finlandia, Alemania o el Reino Unido que muestran porcentajes superiores al $70 \%$, mientras que en España el porcentaje es del $40 \%$ en Bélgica del $18 \%$ y en Italia sólo el $12 \%$. En Suecia, a pesar de la presión legislativa de tener al menos un $25 \%$ de mujeres en los consejos de administración, 2 de las empresas de la muestra les falta todavía representantes femeninos. En

5 No obstante, en Alemania, la mayoría son representantes de empleados. 
Suiza el $50 \%$ de empresas estudiadas no tiene mujeres en sus consejos (60\% en 2003).

\section{CONCLUSIONES}

En este trabajo se ha puesto de manifiesto que existe una preocupación en los distintos países europeos por el tema de la diversidad. Hemos observado que los Códigos de Buen Gobiernos emitidos por los diferentes países tratan la diversidad en mayor o menor grado.

- Casi todos se centran en la diversidad que podríamos denominar no cuantificable: habilidades conocimientos...

- Otros, los menos, además se centran en la diversidad que podríamos denominar cuantificable u observable: género, raza, edad...

- Los Códigos se van renovando, actualizando, y con ello van introduciendo más factores de diversidad.

Encontramos que las prácticas de buen gobierno de las empresas de los países pioneros en la emisión de Códigos de Buen Gobierno son mejores. Los primeros países en elaborar documentos sobre el tema en Europa (Inglaterra $1^{\circ}$, Francia $2^{\circ}$ y Holanda $3^{\circ}$ ) obtienen las mejores calificaciones en el estudio de Heidrick-Struggles (Inglaterra $1^{\circ}$, Holanda $3^{\circ}$ y Francia $4^{\circ}$ ). Sin embargo, Alemania a pesar de haber sido un país que ha emitido documentos relacionados con el tema de forma relativamente temprana tiene una de las últimas calificaciones. Suiza, siendo el último de los países de este estudio en elaborar un Código de Buen Gobierno ha conseguido en 2005 la $2^{a}$ mejor calificación. Suecia también ofrece una disparidad positiva.

No encontramos una relación clara entre la emisión de Códigos de Buen Gobierno y diversidad de género en las empresas de los países que los emiten. Pero sí hemos observado que aquellos países que expresamente recogen en los Códigos la diversidad de género, son los que más la practican en sus empresas (Noruega, Suecia y Finlandia).

Es de notar que Noruega, país que tiene la tasa más alta de presencia femenina en los consejos de administración, muy por encima de la media europea, está obligado por Ley a que exista paridad de género en sus consejos. 
El Código Unificado Español ha apostado por una diversidad de género comparable a estos países. Por tanto, para poder cumplir con estas recomendaciones durante el año 2007, primer informe de gobierno corporativo que se ha de publicar en 2008 , hay mucho que hacer y muchos cambios se han de producir para romper con la realidad actual. Es de esperar que estas recomendaciones, que tanta polémica están suscitando, tengan un efecto positivo a medio o largo plazo en las empresas favoreciendo con ello la eficiencia de las mismas. La certeza y la cercanía en el tiempo de estos logros dependen no sólo de los objetivos establecidos por la propia empresa sino también de las iniciativas gubernamentales relacionas con la Ley Orgánica de Igualdad entre mujeres y hombres.

En este sentido el articulo 75, del título séptimo de la Ley Orgánica de Igualdad aprobado recientemente (marzo 2007) establece que las sociedades procuraran incluir en su consejo de administración un número de mujeres que permita alcanzar una presencia equilibrada de mujeres y hombres en un plazo de ocho años a partir de la entrada en vigor de esta ley. Esto se tendrá en cuenta para los nombramientos que se realicen a medida que venza el mandato de los consejeros designados antes de la entrada en vigor de esta ley.

\section{BIBLIOGRAFÍA}

ADLER, R.D. (2001) "Women in the executive suite correlate to high profits", Working Paper, Pepperdine University.

ALDAMA (2003) Informe de la Comisión Especial para el fomento de la transparencia y seguridad en los mercados $y$ en las sociedades cotizadas. Informe Aldama, 8, enero, 2003.

Alimo-MetCALFE, B. (1995) "An Investigation of Female and Male Constructs of Leadership and Empowerment", Women in Management Review, v.10, 2, pp. 3-8.

BARBERÁ, E.; SARRIó, M.; RAMOS, A. (2000) "Mujeres directivas: promoción profesional en España y el Reino Unido", Quaderns Feministas, $\mathrm{n}^{\circ} 2$, Universidad de Valencia.

BENSCHOP, Y. (2001) "Pride, prejudice and performance: relations between HRM, diversity and performance", International Journal of Human Resource Management, 12 (7), pp. 1.166-1.181. 
BONNAFOUS-BOUCHER, M. (2005) "From Government to Governance", Ethical Perspective, 12/4 December 2005, pp. 521-534.

BuRgess, Z. \& THARENOU, P. (2002) "Women board directors, Characteristics of the few", Journal of Business Ethics, 37, pp. 39-49.

CARTER, D.A.; SIMKINS, B.J.; GARY SIMPSON, W. (2003) "Corporate governance, board diversity and firm value", Financial Review, vol. 38, pp. 33-53.

CATALYST (2004) The Bottom line: Connecting corporate performance and gender diversity. NY: Catalyst.

CE (2004) Recomendación (913/2004/CE), de 14 de diciembre, sobre la promoción de un régimen adecuado de retribución a los administradores de las sociedades cotizadas.

- (2005) Recomendación (162/2005/CE), de 15 de febrero, sobre el papel de los consejeros externos o supervisores de las sociedades cotizadas y sobre los comités del consejo de administración o supervisión.

CNMV (2005) Informe de Gobierno Corporativo de las Entidades Emisoras de Valores Admitidos a Negociación en Mercados Secundarios Oficiales. Diciembre 2005.

- (2006a) Proyecto de Código Unificado de Recomendaciones sobre buen Gobierno de las Sociedades Cotizadas.18 Enero 2006.

- (2006b) Informe del Grupo Especial de Trabajo sobre Buen Gobierno de las Sociedades Cotizadas. Código Unificado de Buen Gobierno.19 Mayo 2006.

EPWN (2004) European Board Women Monitor 2004, European Professional Women's Network \& Egon Zehnder International.

FARREL, K.A. y HIRSCH, P.L. (2005) "Additions to corporate boards: the effect of gender", Journal of Corporate Finance, Vol. 11, pp. 85-106.

FÉlez, M.; Romero, A.; PueYo, R. y Llaría, J. (2004) "Género y los Consejos de Administración de las Empresas del IBEX 35". Documento de Trabajo de la Fundación Ecológica y Desarrollo, abril 2004.

GómEZ ANSón, S. (2005) "Diversidad de Género en los Consejos de Administración de las Sociedades Cotizadas y Cajas de Ahorros Españolas", Papeles de la Fundación de Estudios Financieros, 12.

GREGORY, H.J. (2002) Comparative Study of Corporate Governance Codes Relevant to the European Union and Its Member States. Weil, Goatshal $\&$ Manges. On behalf of the European Commission.

HART, S. y MILTEIN, M. (2003) "Creative Sustainable Value", Academy of Management Executive, 17(2), pp. 56-67. 
HEIDRICK \& STRUGGLES (2005) Corporate governance in Europe: what's the Outlook?. H \& S Inc.

HIGGS, D. (2003) The Higgs Report: Review of role and effectiveness of non-executive directors. UK: The Department of Trade and Industry, January.

Jimeno, F.J.; Redondo, M. (2005) "Sobre el techo de Cristal Empresarial", AECA. Revista de la Asociación española de Contabilidad y Administración de Empresas, 71, pp. 42-46.

KANDOLA, R.S.; FULLERTON, J. (1998) Diversity in action: managing the mosaic. Institute of Personnel and Development.

LEY ORGÁNICA DE IGUALDAD (2007) Ley Orgánica de Igualdad 3/2007, de 22 de marzo para la igualdad efectiva de mujeres y hombres.

LLOPIS RIVAS, A.M. (2006) "Nuevo Código Unificado de Buen Gobierno. La importancia de los independientes y la incorporación de las mujeres. España entre los primeros de Europa en 'governance"', AECA, 76, Septiembre, pp. 56-60.

MCDOUGLAS, M. (1996) "Equal opportunities versus managing diversity another challenger for Public Sector Management?", International Journal Public Management, 9, pp. 62-72.

MONACCI, M. (1997) Genere e organizzazione. Questioni e modelli interpretativi. Milan: Gurini e Associati.

OECD (2004) Organización para la Cooperación y el Desarrollo Económicos. Principios de Gobierno Corporativo de la OCDE. Ministerio de Economía y Hacienda.

OHLOTT, R.; Ruderman, M.N.; MCCAUley, C.D. (1994) "Gender Differences in Managers", Developmental Job Experiences. Academy of management Journal, 37, pp. 46-67.

OLIVENCIA (1998) Documento de la Comisión Especial para el estudio de un código ético de los consejos de administración de las sociedades: el gobierno de las sociedades cotizadas. Informe Olivencia. 26 de febrero. Madrid.

Pounder, J.S.; Coleman, M. (2002) "Women better leaders that men? In general and educational management it still all depends". Leadership \& \& Organization Development Journal, 23/3, pp. 122-133.

Powell, G.N.; ButTerfield, D.A. (1994) "Investigating the 'Glass Ceiling' Phenomenon: An Empirical Study of Actual Promotions to top Management", Academy of Management Journal, vol. 37, 1, Feb, pp. 68-86. 
RAGINS, B.R.; TOWNSEND, B.; MATTIS, M. (1998) Gender gap in the executive suite: CEOS and Female executives report on breaking the glass ceiling. Academy of Management Executive.

RoBInSON, G. y DECHANT, K. (1997) "Building a Business Case for Diversity", The Academy of Management Executive, vol. 11(3), pp. 21-31.

RoSENZWEIG, P. (1998) "Managing the New Global Workforce: Fostering Diversity, Forging Consistency", European Management Journal, 16, pp. 644-652.

TYSON, L. (2003) The Tyson report on the recruitment and development of non-executive directors. London Business School, July.

UE (2002) "Report of the High Level Group of Company Experts on a Modern Regulatory Framework for Company Law in Europe", November.

WIRTH, L. (2002) Romper el techo de cristal. Las mujeres en puestos de dirección. Colección informes OIT $\mathrm{n}^{\circ} 58$, Ministerio de Trabajo y Asuntos Sociales. 